\title{
New Results on Jet Fragmentation at CDF
}

\author{
Sergo Jindariani, on behalf of the CDF collaboration \\ Department of Physics, University of Florida, Gainesville, FL 32611, USA
}

Received on 1 November, 2006

\begin{abstract}
Presented are the latest results of jet fragmentation studies at the Tevatron using the CDF Run II detector. Studies include the distribution of transverse momenta $(\mathrm{Kt})$ of particles jets, two-particle momentum correlations, and indirectly global event shapes in $p \bar{p}$ collisions. Results are discussed within the context of recent Next-to-Leading Log calculations as well as earlier experimental results from the Tevatron and $e^{+} e^{-}$colliders.
\end{abstract}

Keywords: QCD; Fragmentation; Correlation; Transverse Momenta; Event Shapes

\section{INTRODUCTION}

The evolution of jets is driven by the emission of gluons at very small transverse momentum with respect to the jet axis. Analytical predictions are based on Next-to-Leading Log Approximation (NLLA) [1] calculations supplemented with the hypothesis of Local Parton-Hadron Duality (LPHD) [2]. NLLA provides an analytical description of parton shower formation, while LPHD states that the hadronization process takes place locally, and, therefore, properties of hadrons and partons at the end of the parton shower are closely related. Detailed studies of jet fragmentation allow one to better understand the relative roles of perturbative parton showering and non-perturbative hadronization in shaping the main jet characteristics. Past experimental studies of inclusive distributions of particles in jets [3][4] have shown good agreement with theoretical predictions, suggesting that the perturbative QCD (pQCD) stage of jet formation must be dominant, and the role of the non-perturbative stage is reduced to converting final partons into hadrons without significantly affecting the multiplicities and momenta.

In this paper we give an overview of recent fragmentation measurements made at CDF and discuss the results within the context of recent analytical calculations.

\section{TWO-PARTICLE MOMENTUM CORRELATIONS}

The results of the measurement of the two-particle momentum correlations in jets are based on dijet data collected during the running period from February 2002 to August 2004 (approximately $400 \mathrm{pb}^{-1}$ of data). The analysis was carried out in the dijet center-of-mass frame. Data were divided into seven dijet mass bins with the average invariant mass $M_{j j}=76,108$, $148,200,270,360$ and $475 \mathrm{GeV}$. The correlation function was introduced in terms of the parameter $\xi=\ln \left(E_{\text {jet }} / p_{\text {hadron }}\right)$ and was defined as a ratio of two- and one-particle inclusive momentum distributions:

$$
C\left(\Delta \xi_{1}, \Delta \xi_{2}\right)=\frac{D\left(\xi_{1}, \xi_{2}\right)}{D\left(\xi_{1}\right) D\left(\xi_{2}\right)},
$$

where both inclusive distributions $D(\xi)=\frac{d n}{d \xi}$ and $D\left(\xi_{1}, \xi_{2}\right)=$ $\frac{d^{2} n}{d \xi_{1} d \xi_{2}}$ were normalized to unity. The results were obtained for charged particles within a restricted cone with an opening angle of $\theta_{c}=0.5$ radians around the jet axis. The underlying event contribution was subtracted using the complementary cones technique[4].

In NLLA [5] the correlation function $C\left(\Delta \xi_{1}, \Delta \xi_{2}\right)$ is predicted to be:

$$
C\left(\Delta \xi_{1}, \Delta \xi_{2}\right)=c_{0}+c_{1}\left(\Delta \xi_{1}+\Delta \xi_{2}\right)+c_{2}\left(\Delta \xi_{1}-\Delta \xi_{2}\right)^{2},
$$

where the parameters $c_{0}, c_{1}$ and $c_{2}$ define the strength of the correlation and are functions of the so called "jet hardness" $\left(Q=E_{j e t} \theta_{c}\right)$ and the parton shower cutoff scale $Q_{e f f}$. $\Delta \xi=\xi-\xi_{0}$, where $\xi_{0}$ corresponds to the peak of the inclusive momentum distribution $D(\xi)$.

Fig. 1 shows diagonal profiles of the correlation distribution for dijet mass bin with $Q=50 \mathrm{GeV}$. The solid line corresponds to the NLLA fits according to the Eq. 2 with three free parameters $c_{0}, c_{1}$ and $c_{2}$. The dashed line corresponds to the analytical prediction for the value of $Q_{e f f}=230 \mathrm{MeV}$, extracted from the fits of the inclusive momentum distributions. The overall qualitative agreement between data and NLLA calculation results is very good, data follows theoretical trends. However, a small offset in the overall level of correlation is observed in all seven dijet mass bins, indicating that theory overestimates parameter $c_{0}$ of the correlation.

Fig. 2 shows evolution of correlation parameters $c_{1}$ and $c_{2}$ with jet hardness. Each data point corresponds to the value of the parameter obtained in particular dijet mass bin. CDF data points were fit to the analytical NLLA function, while parton shower cutoff $Q_{e f f}$ was treated as a free parameter. The value of $Q_{e f f}$ obtained from the fit of $c_{1}$ was $147 \pm 10$ (stat $) \pm$ 79 (syst) $\pm 2(p d f) \mathrm{MeV}$. The value of $Q_{\text {eff }}$ obtained from the fit of $c_{2}$ was $131 \pm 12($ stat $) \pm 86($ syst $) \pm 3(p d f) \mathrm{MeV}$.

The comparison to Monte-Carlo revealed that both Pythia Tune A and Herwig 6.5 reproduce correlation in data fairly well.

\section{III. $k_{T}$ DISTRIBUTIONS}

The measurement of intrinsic $k_{T}$ (transverse momenta of particles with respect to jet axis) distributions is of particular interest, not only because of the recent theoretical work [6], but also because it allows to probe softer particle spectra than previously measured observables. The CDF measurement is based on approximately $1 \mathrm{fb}^{-1}$ of data. Same event selection 

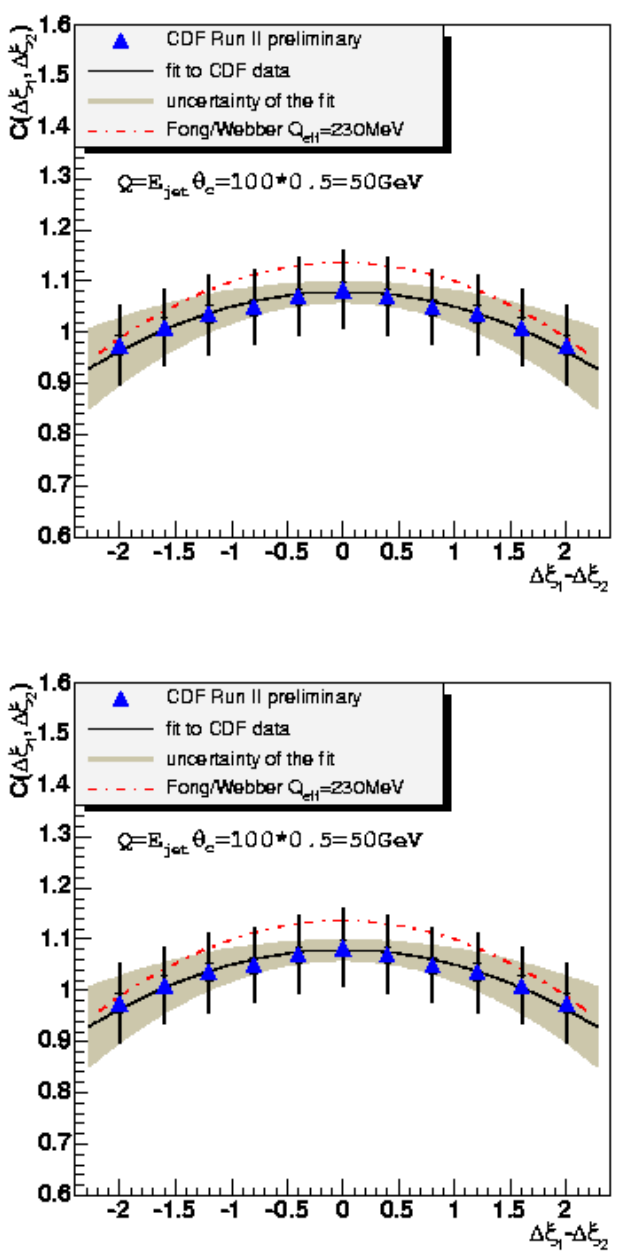

FIG. 1: Central diagonal profiles $\Delta \xi_{1}=-\Delta \xi_{2}$ and $\Delta \xi_{1}=\Delta \xi_{2}$ of the correlation distribution $C\left(\Delta \xi_{1}, \Delta \xi_{2}\right)$

criteria, as described in the previous section, was applied.

In [6] theoretical predictions were made in the framework of the Modified Leading Log Approximation (MLLA). Distributions were normalized to the average multiplicity of particles in jets. However, past CDF studies indicated that the multiplicity of particles at CDF agrees well with the MLLA predictions. The goal of this study was to compare the shape of the $k_{T}$ distrubutions rather than the overall normalization, therefore the distributions were normalized to the number of entries in the bin $-0.2<\ln \left(k_{T}\right)<0.0$.

Fig.3 shows $k_{T}$ distributions in data on top of the MLLA curves for two different values of jet hardness $Q$. One can see that at large values of $\ln \left(k_{T}\right)$ theory predicts more particles than observed in data. However, one should keep in mind that MLLA calculations were carried out in the regime of soft approximation, i.e. $p_{\text {hadron }} / E_{j e t} \ll 1$. This translates into the condition for $k_{T}: \ln \left(k_{T} / Q_{e f f}\right)<\ln \left(k_{T} / Q_{e f f}\right)-2.5$, therefore settting the upper boundary on the validity of MLLA predictions. Within the validity region, the agreement between data
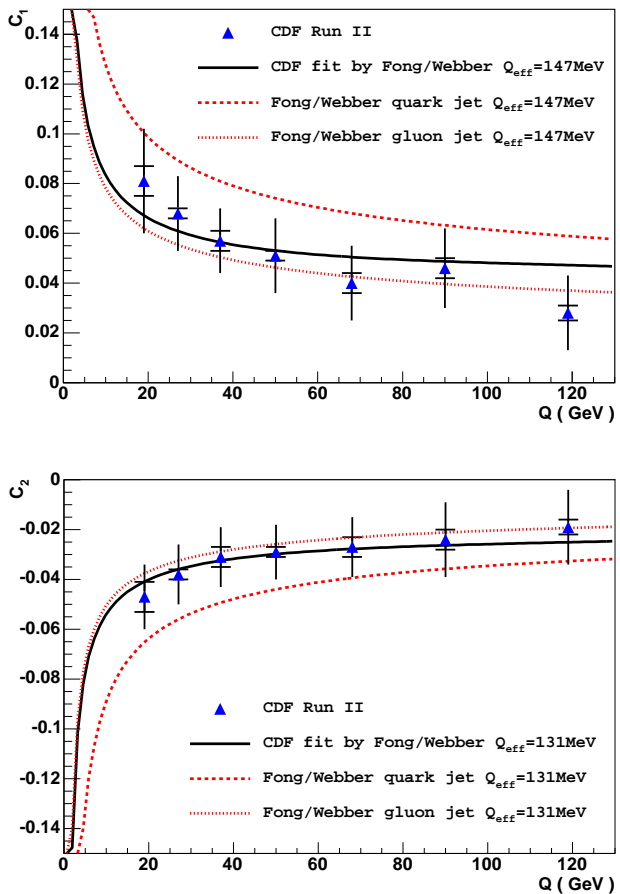

FIG. 2: The evolution of correlation parameters $c_{1}$ and $c_{2}$ with "jet hardness".

and theory is fairly good, suggesting that the $k_{T}$ distributions do not change significantly in the process of hadronization.

\section{INDIRECTLY GLOBAL EVENT SHAPES}

In the broadest terms, event shapes provide a means of understanding the energy flow in hadronic final states. Their sensitivity to both perturbative and non-perturbative aspects of QCD makes them an important addition to the study of jet fragmentation. Of particular interest on the non-perturbative front is the study of QCD power-corrections within the Dokshitzer-Marchesini-Weber (DMW) approach [7], which introduces the notion of an infrared finite coupling. Following a dispersive approach, the model predicts a shift in the perturbatively calculated event shape distributions. The study of power corrections has found success in data from $e^{+} e^{-}$and Deep Inelastic Scattering (DIS) experiments, but has yet to find footing in a hadron collider environment.

As motivated by theory [8], events are selected with at least two jets in the central region $\eta_{j e t}<0.7$. In addition, a cut is placed on the transverse energy of the leading jet $E_{T}^{\text {jet } 1}>50 \mathrm{GeV}$. The measurement is then performed using calorimeter towers within the region $|\eta|<3.0$. The CDF collaboration is actively pursuing the full measurement of six complementary indirectly global event shape observables introduced in [7]. Amongst these, is the Transverse Thrust Minor, which is defined as 

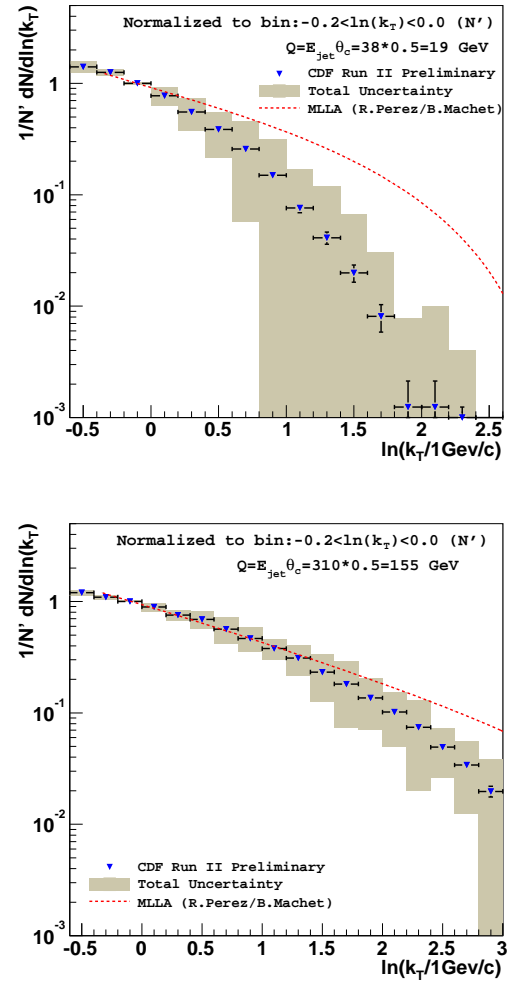

FIG. 3: $\ln \left(k_{T}\right)$ distributions for two dijet mass bins with $\mathrm{Q}=19$ and $155 \mathrm{GeV}$. CDF data is compared to the MLLA predictions.

$$
T_{m, \perp} \equiv \frac{\sum_{i}\left|\vec{q}_{\perp, i} \times \vec{n}_{T}\right|}{\sum_{i}\left|\vec{q}_{\perp i}\right|}
$$

where $\vec{n}_{T}$ is the transverse thrust axis and is defined as the direction which maximizes the quantity: $T_{\perp} \equiv \max _{\vec{n}_{T}} \frac{\sum_{i}\left|\vec{q}_{\perp \cdot} \cdot \vec{n}_{T}\right|}{\sum_{i}\left|\vec{q}_{\perp i}\right|}$.

Figure 4 shows between data and Pythia Tune A Monte Carlo (MC) with CDF II detector simulation for the transverse thrust minor. The plot reveals a very prominent shift in the data away from the 2-jet region with respect to the MC. Preliminary studies have shown that there are no significant detector corrections in unfolding back to the particle level. At the time of this conference, we are awaiting perturbative predictions (Next-to-Leading-Log matched to Next-to-Leading-
Order) for these observables as well as predictions for the nonperturbative corrections.

\section{CONCLUSIONS}

CDF continues its extensive jet fragmentation program, which includes recent measurements of the two-particle momentum correlations and intrinsic $k_{T}$ distributions. The data was compared to the NLLA calculations combined with the hypothesis of Local Parton-Hadron Duality. It has been shown that the two-particle momentum correlations do survive hadronization. The average extracted value of the parton

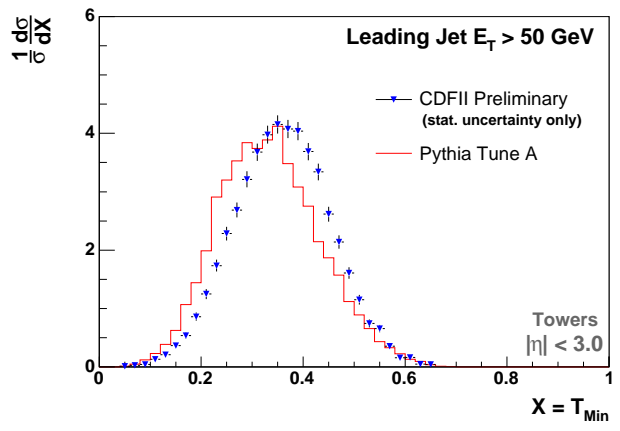

FIG. 4: Comparison of Data and Pythia Tune A for Transverse Thrust Minor.

shower cutoff scale was $139 \pm 11($ stat $) \pm 84($ syst $) \pm 3(p d f s)$ $\mathrm{MeV}$ and appears to be reasonable. The measurement of the $k_{T}$ distributions showed good agreement between CDF data and MLLA within the validity range of the theoretical predictions. The results of these two measurements give further support to the hypothesis of LPHD.

Finally, the first measurement of indirectly global event shapes in hadron collisions is underway at CDF. Preliminary results have shown that data is shifted away from the two jet region with respect to the predictions of Pythia Monte Carlo. At e+e- and DIS experiments a similar shift was observed and accounted for by $1 / Q$ non-perturbative corrections to QCD.

\section{Acknowledgements}

Special thanks to L. Pinera and A. Korytov for their help in the preparation of these proceedings.
[1] Yu. Dokshitzer, V. Khoze, A. Mueller, and S. Troyan, Basics of Perturbative QCD, edited by J. Tran Thanh Van (Editions Frontières, Gif-sur-Yvette, 1991);

[2] Ya.I. Azimov, Yu. Dokshitzer, V. Khoze, and S. Troyan, Z. Phys. C 27, 65 (1985); 31, 213 (1986);

[3] CDF Collab., T.Affolder et al., Phys. Rev. Lett. 87, 211804 (2001).
[4] CDF Collab., T.Acosta et al., Phys. Rev. D 68, 012003 (2003).

[5] C. Fong and B.R. Webber, Nucl. Phys. B 355, 54 (1991)

[6] R. Perez-Ramos, B. Machet, JHEP 04, 043 (2006).

[7] A.Banfi, G. Salam, and G. Zanderighi, JHEP 0408, 062 (2004).

[8] Y.Dokshitzer, G.Marchesini, and B.R.Webber, Nucl. Phys. B 469, 93 (1996). 